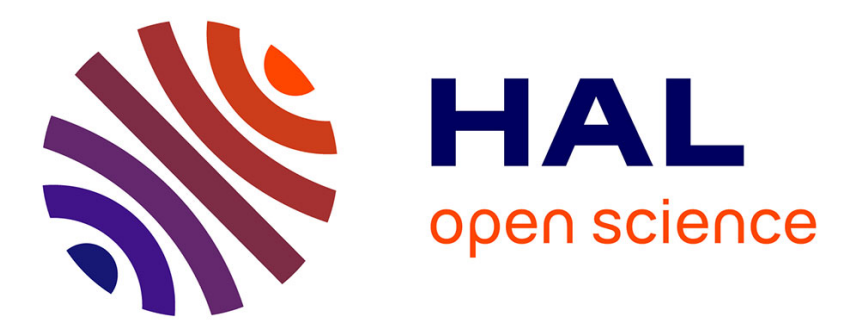

\title{
The Darcytron: a pressure-imposed device to probe the frictional transition in shear-thickening suspensions
}

Cécile Clavaud, Bloen Metzger, Yoel Forterre

\section{To cite this version:}

Cécile Clavaud, Bloen Metzger, Yoel Forterre. The Darcytron: a pressure-imposed device to probe the frictional transition in shear-thickening suspensions. Journal of Rheology, 2020, 64 (2), pp.395 403. 10.1122/1.5135370 . hal-02351663v2

\section{HAL Id: hal-02351663 \\ https://hal.science/hal-02351663v2}

Submitted on 8 Jan 2021

HAL is a multi-disciplinary open access archive for the deposit and dissemination of scientific research documents, whether they are published or not. The documents may come from teaching and research institutions in France or abroad, or from public or private research centers.
L'archive ouverte pluridisciplinaire HAL, est destinée au dépôt et à la diffusion de documents scientifiques de niveau recherche, publiés ou non, émanant des établissements d'enseignement et de recherche français ou étrangers, des laboratoires publics ou privés. 


\title{
The Darcytron: a pressure-imposed device to probe the frictional transition in shear-thickening suspensions
}

\author{
Cécile Clavaud, ${ }^{\text {a) }}$ Bloen Metzger, ${ }^{\text {b) }}$ and Yoël Forterrec) \\ ${ }^{1}$ Aix Marseille Univ, CNRS, Institut Universitaire des Systèmes Thermiques et Industriels, 13453 Marseille, \\ France.
}

(Dated: 7 February 2020)

\begin{abstract}
In this paper, we present a new device called the Darcytron, allowing pressure-imposed rheological measurements on dense suspensions made of very small particles, like shear-thickening suspensions. The main idea is to impose and control the particle pressure using a vertical Darcy flow across the settled bed of particles. We first validate the proof of concept of the Darcytron on a standard (non shear-thickening) suspension composed of large glass particles. We then use this new device to investigate the frictional behavior of a model shear-thickening suspension composed of small silica particles. These results provide direct evidence of a transition between a frictionless and a frictional state as the particle pressure is increased, providing support to the recent frictional transition scenario for shear thickening.
\end{abstract}

\section{INTRODUCTION}

The dramatic viscosity increase in some fluid/particules mixtures above a critical shear rate, called shear-thickening, is one of the most intriguing phenomenon observed in dense particulate suspensions ${ }^{1-3}$. Shear thickening occurs in suspensions composed of sufficiently small particles, typically below $50 \mu \mathrm{m}$, and appears in many practical cases such as concrete, chocolate formulation or body armors design ${ }^{4-6}$. In spite of this widespread nature, shear thickening has long remained poorly understood.

Recently, discrete numerical simulations ${ }^{7,8}$ together with a theoretical model $^{9}$ have proposed that shear-thickening results from a frictional transition. In suspensions composed of large and rigid grains, which interact solely through solid Coulombian frictional contacts and hydrodynamic forces (called standard suspension in the following), the rheology is expected to be rate independent, i.e. Newtonian. The model and the simulations thus considered the fact that in shear-thickening suspensions, the grains are small enough that electrostatic charges or a polymer coating at their surface can generate a significant repulsive force between them. Introducing this additional force scale in the description has a major impact on the suspension rheology: as shown by both the simulations and the model, this additional repulsive force induces the possibility of a frictionless state of the suspension. Under low stress, the interparticle force repels particles and thus prevents solid frictional contacts between them. As a result, particles within the sheared suspension can slide easily against each other without friction, yielding a suspension of low viscosity. Under large stress however, the repulsive force becomes negligible and solid frictional contact can occur: the suspension then recovers a standard frictional behavior of larger viscosity.

Encouraging support for this scenario has already been provided by very recent experiments. For instance, using a specific AFM technique ${ }^{10}$, Comtet et al. (2017) directly mea-

\footnotetext{
a)Electronic mail: cecileclavaud@free.fr

b)Electronic mail: bloen.metzger@univ-amu.fr

c)Electronic mail: yoel.forterre@univ-amu.fr
}

sured the microscopic friction coefficient $\mu_{p}$ between two grains of a shear-thickening suspension. They showed that such grains under low stress are indeed quasi-frictionless with $\mu_{p} \approx 0.02$, and become frictional above a critical normal load, with $\mu_{p} \approx 0.45$. This frictional behavior, observed here at the particle scale, corresponds to that expected in the framework of the frictional transition model. At the flow scale conversely, supporting evidences are generally less direct. Experiments showed results consistent with the model ${ }^{11,12}$ but no direct evidence of a frictional transition could be highlighted. The main difficulty lies in that these experiments were carried out using standard rheological techniques, where the particulate volume fraction is fixed. Such an approach provides access to the suspension viscosity, shear stress and shear rate, but it gives no information about the frictional properties of the suspension - information that is essential in order to probe the frictional transition model.

To access the suspension frictional properties, we proposed in a previous article a different approach where, instead of imposing the volume fraction, the particle pressure was imposed (Clavaud et. al $(2017)^{13}$ ). One major virtue of imposing the particle pressure is that the rheological law then naturally takes the form of a friction law defining the macroscopic suspension friction coefficient of the suspension $\mu=\tau / P_{p}$ as the ratio of the shear stress $\tau$ and to the particle normal pressure $P_{p}$. In Clavaud et al. (2017), we studied the quasi-static avalanche angle $\theta$ of a pile of non-buoyant repulsive particles immersed in water in a slowly rotating drum. In this configuration, both the shear stress and the particle pressure are fixed and imposed by the weight of the flowing layer of grains at the top of the pile. Consequently, the frictional state of the suspension can be directly inferred from the avalanche angle since $\mu=\tan \theta$. By tuning the range of the repulsive force between particles, these experiments revealed the expected frictional transition. Moreover, they showed that shear thickening relies on the existence of a frictionless state of the suspension under low stress.

These results obtained using a rotating drum configuration strongly support the frictional transition scenario. However, in such a configuration, the particle pressure cannot be adjusted and the frictional transition could only be observed by tuning the physical and chemical properties of the suspen- 


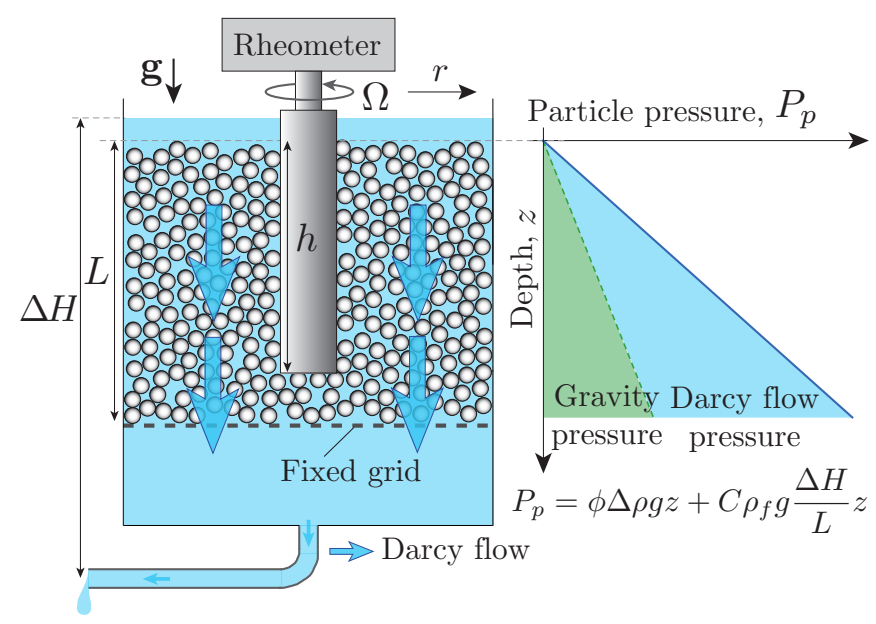

FIG. 1. The Darcytron: general concept. The granular pressure $P_{p}$ is controlled by imposing a vertical Darcy flow across the settled bed of particles.

sion. To fully explore this frictional transition, it is now essential to vary the particle pressure. This represents a real experimental challenge, since it requires the development of an original rheometer able to perform pressure-imposed rheological measurements on shear-thickening suspensions, which are composed of very small particles (below $50 \mu \mathrm{m}$ ). Existing pressure-imposed rheometers use a moving grid to apply the desired normal stress on the particles ${ }^{14-16}$. They are thus adapted to suspensions composed of large particles (typically larger than $300 \mu \mathrm{m}$ ) but inappropriate for studying shearthickening suspensions.

In this paper, we develop a new pressure-imposed rheological device, the Darcytron, based on an original concept: the idea here is to impose the particle pressure, not by using a moving grid like in Boyer et al. rheometer ${ }^{14}$, but by imposing a Darcy flow across the suspension. As we will show, this method is crucial to extend pressure-imposed measurements to shear-thickening suspensions given their small particle size. The paper is organized as follows: we first present the principle of the Darcytron. We then validate its concept by investigating the frictional behavior of a standard (non shearthickening) suspension composed of large glass particles. Finally, we present the results highlighting the frictional transition when varying the particle pressure in a model shearthickening suspension composed of small silica particles.

\section{THE DARCYTRON: GENERAL CONCEPT}

A sketch of the Darcytron is represented in figure 1. The configuration is a wide gap annular shear flow with a suspension of non-buoyant particles immersed in a Newtonian fluid of viscosity $\eta_{f}$. In this configuration, particles settle at the bottom of the cell and the vertical particle stress $\sigma_{z z}^{p} \equiv P_{p}$, called particle pressure in the following, is initially the confining pressure imposed by gravity, $P_{p}(z)=\phi \Delta \rho g z$, where $\phi$ is the volume fraction of the pile, $\Delta \rho=\rho_{p}-\rho_{f}$ the density difference between the particles and the fluid, $g$ the gravity and $z$ the vertical position measured relatively to the sediment free surface. The key idea of the Darcytron is to control the particle pressure $P_{p}$ by imposing, in addition to gravity, a vertical Darcy flow through the settled pile of grains. To this end, the wall at the bottom of the grain pile is made out of a fixed porous grid with holes smaller than the particle size. The Darcy flow is imposed by setting a height difference $\Delta H$ between the fluid free surface and the tube outlet connected to the bottom of the cell. In this case, the flow inside the granular sediment is vertical and the iso-pressure levels are horizontal (r-independent), even in the presence of slight horizontal gradients of volume fraction. When the Darcy flow is turned on, the total vertical stress acting on the particles is now given by the sum of both the confining pressure due to gravity and the Darcy flow pressure. The vertical momentum balance on the particle phase yields ${ }^{17,18}$

$$
P_{p}(z)=\phi \Delta \rho g z+\nabla P_{f} z
$$

where $\nabla P_{f}=C \rho_{f} g \Delta H / L$ is the Darcy pressure gradient imposed across the granular bed, $L$ is the height of the granular bed and $C$ is a numerical constant (smaller than 1 ) accounting for the pressure loss in the grid. Everything thus happens as if the grains were submitted to an effective gravity

$$
g_{\text {eff }}=g\left(1+\frac{C \rho_{f} \Delta H}{\phi \Delta \rho L}\right),
$$

and the particle pressure can simply be recasted in

$$
P_{p}(z)=\phi \Delta \rho g_{\text {eff }} .
$$

Therefore, in this set-up, by controlling the height of the water outlet $\Delta H$, one can easily vary the effective gravity and thereby the pressure $P_{p}$ acting on the particles. Another important advantage of this configuration is that the bottom porous grid is fixed and sealed to the side walls, unlike Boyer's configuration where the grid is mobile. Suspensions composed of much smaller particle can thus be investigated without particle leakage issues.

The inner cylinder is driven by a rheometer head which measures the torque $\Gamma$ required to maintain a constant rotation speed $\Omega$. This torque can be computed as $\Gamma=$ $2 \pi a^{2} \int_{0}^{h} \sigma_{r \theta}(z) d z$, where $a$ is the radius of the inner cylinder, $h$ is the cylinder depth immersed within the sediment and $\sigma_{r \theta}$ is the total shear stress exerted on the inner cylinder in the azymuthal direction $\theta$. By definition the friction coefficient of the suspension $\mu$ is the ratio of the shear stress to the normal particle stress ${ }^{14,19}, \mu=\sigma_{r \theta} / \sigma_{r r}^{p}$. Moreover, since $\sigma_{r r}^{p}=K \times P_{p}$ where $K$ is a numerical constant accounting for the stress anisotropy within the granular phase, we obtain $\Gamma=\mu \times K \pi a^{2} \phi \Delta \rho g_{\text {eff }} h^{2}$. Measuring the torque thereby provides direct access to the suspension friction coefficient

$$
\mu=\frac{\Gamma}{K \pi a^{2} \phi \Delta \rho g_{\mathrm{eff}} h^{2}} .
$$

Computing $\mu$ from the above formula requires the two numerical constants $C$ (accounting for the grid pressure drop) 

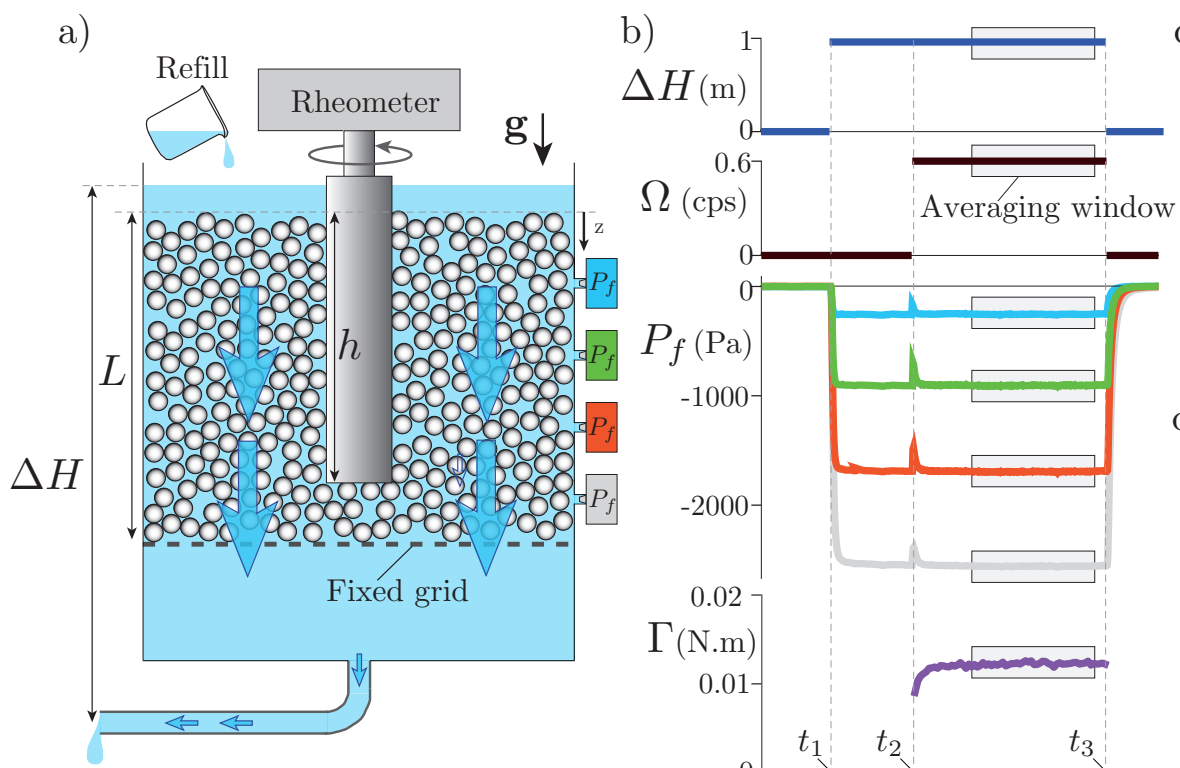

c)
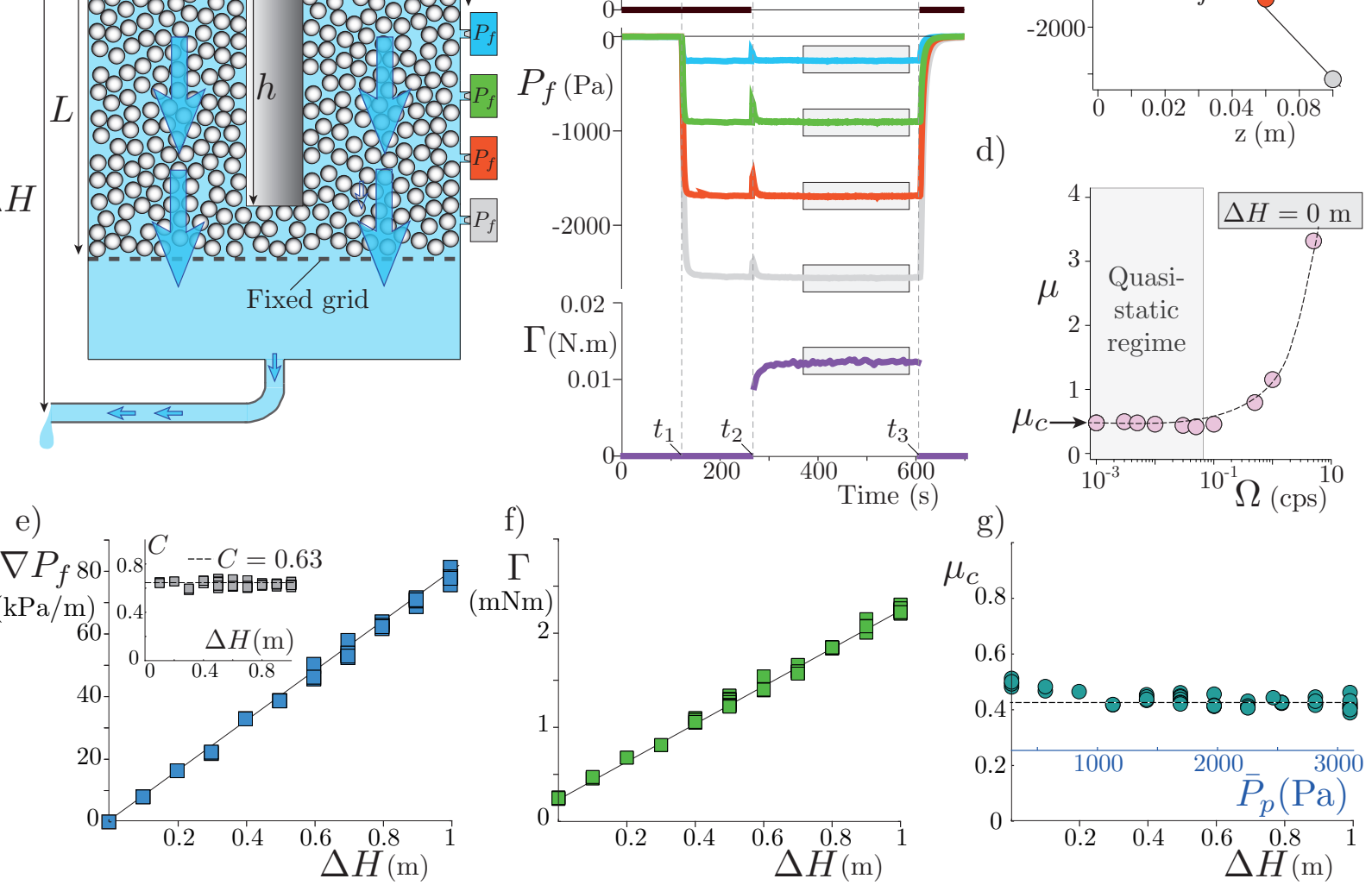

FIG. 2. The Darcytron: proof of concept with a standard (non shear-thickening) suspension (glass beads $d \approx 500 \mu$ m immersed in a Newtonian liquid of viscosity $\eta_{f}=0.4 \mathrm{~Pa} \mathrm{~s}$ ). a) Detailed set-up with the differential pressure sensors. b) Height of the water outlet $\Delta H$, rotation speed $\Omega$, liquid pressures $P_{f}$ measured at successive heights $z$ and torque $\Gamma$ versus time for a typical measurement. c) Time averaged fluid pressures $P_{f}$ measured at various heights $z$ give access to the Darcy pressure gradient $\nabla P_{f}$ and thus to $C=-\nabla P_{f} L / \rho_{f} g \Delta H$, see Equ. 5. d) Suspension friction coefficient $\mu$ versus rotation speed $\Omega$ (cps: cycle per second). The grey area indicates the quasi-static regime. e) Darcy pressure gradient $\nabla P_{f}$ versus $\Delta H$. Inset: Corresponding values of $C$ computed using Eqn. 5. f) Torque $\Gamma$ versus $\Delta H$. g) Quasi-static friction coefficient $\mu_{c}$ versus $\Delta H$ and also versus the average granular pressure $\bar{P}_{p}$ (blue axes) obtained using $C=0.63$.

and $K$ (accounting for the stress anisotropy) to be set. In their work on the rheology of dense suspensions, Dbouk et $a l .{ }^{20}$ report that the particle normal stress in the gradient direction (here along $r$ ) is twice that in the vorticity direction (here along $z$, direction along which the particle pressure is imposed) such that $K=2$. However, they addressed suspensions of volume fractions ranging from 20 to $48 \%$. In our experiments, we will focus on the frictional behavior of the suspension in the quasi-static regime, where $\phi \rightarrow \phi_{c}$. We will therefore use the anisotropy reported from experiments performed with dense granular systems ${ }^{21}$ for which $K \approx 1$. The value of $C$ will be discussed and measured in the next section.

We have described how the Darcytron enables to control the particle pressure $P_{p}$ and probe the friction coefficient $\mu$ of the suspension. In the following, measurements will be performed at very low rotation speed $\Omega \rightarrow 0$ for which, under imposed particle pressure, the viscous number $J=\eta_{f} \dot{\gamma} / P_{p} \rightarrow 0$ and the suspension volume fraction $\phi \rightarrow \phi_{c}$, where $\phi_{c}$ is the maximum packing fraction of the suspension under steady shear. In this regime, the particulate flow is localized close to the cylinder within a thin shear band of few particle diameters width in the $(r, \theta)$ plane $^{22}$. Carrying out measurements within this so-called quasi-static or jamming limit is particularly advantageous. First, in this regime the suspension friction coefficient $\mu(J \rightarrow 0) \equiv \mu_{c}$ is a well-documented monotonic function of the interparticle friction coefficient $\mu_{p}{ }^{19,23,24}$. Such measurements will therefore give us the opportunity to directly probe whether the frictional behavior of the particles changes when the confining pressure is varied, as predicted by the frictional transition model. Second, as $\phi \rightarrow \phi_{c}$, the 
sample will remain homogeneous during measurements as no migration of the particles can take place.

\section{PROOF OF CONCEPT WITH A STANDARD (NON SHEAR-THICKENING) SUSPENSION}

Before addressing the frictional behavior of shearthickening suspensions, we first validated the Darcytron concept on a standard (non shear-thickening) suspension of macroscopic $(d \approx 500 \mu \mathrm{m})$ frictional particles. In this case, all colloïdal forces, such as Brownian or short range repulsive forces, are completely negligible relative to the external imposed stress, such as gravity or Darcy pressure. The interparticle solid friction $\mu_{p}$ and thus the quasi-static suspension friction coefficient $\mu_{c}$ are then expected to be constant, i.e. independent of the confining pressure $P_{p}$.

\section{A. Detailed set-up and protocol}

A more detailed sketch of the Darcytron is provided in Figure 2.a. The cell is made out of transparent PMMA plates of height $12.5 \mathrm{~cm}$ and width $10 \times 10 \mathrm{~cm}$. The particles are large glass spherical beads (diameter $d=487 \pm 72 \mu \mathrm{m}$ and density $\rho_{p}=2500 \mathrm{~kg} / \mathrm{m}^{3}$ ) immersed in a viscous and Newtonian mixture of Ucon oil and water of viscosity $\eta_{f}=0.4 \mathrm{~Pa}$ s and density $\rho_{f}=1005 \mathrm{~kg} / \mathrm{m}^{3}$. The fluid is thoroughly degassed using a vacuum pump. The particles are cleaned using ultrasounds and deionized water and then placed into the cell to form sediment of height $L=8.4 \mathrm{~cm}$.

The inner cylinder is a roughened metallic rod of radius $a=6.2 \mathrm{~mm}$ immersed in the granular bed to a depth of $h=7.1$ $\mathrm{cm}$ and driven at constant rotation speed $\Omega$ by an Anton Paar MCR 501 rheometer. The grid at the bottom of the cell is made out of a filter cloth sandwiched between two metallic grids. The cloth has $220 \mu \mathrm{m}$ holes, while those of the metallic grids are $3 \mathrm{~mm}$ large. The tube at the bottom of the cell, which allows us to control the height of the water outlet $\Delta H$, has a large diameter of $10 \mathrm{~mm}$ to minimize the viscous pressure drop within the tube. A valve (not sketched) is mounted at the tube end to turn on the Darcy flow across the settled bed of particles. In order to keep $\Delta H$ (which controls the particle pressure) constant during an experiment, the cell is refilled with the suspending fluid as sketched in Figure 2.a. Four differential pressure sensors are mounted on one of the side walls to measure the fluid pore-pressure $P_{f}$ within the sediment, see Figure 2.a. This measurement is done following the same technique as Dbouk et al. ${ }^{20}$.

An example of measurement is shown in Figure 2.b. At $t=0$, the valve is closed (no Darcy flow, $\Delta H=0$ ) and the inner cylinder is at rest $(\Omega=0)$. In such a configuration, which serves as a reference, we set the differential pressure sensor to zero to remove the contribution of the hydrostatic pressure and of any sensor offset. At $t=t_{1}$, the valve is opened $(\Delta H=1 \mathrm{~m})$ to initiate the Darcy flow: a stationary fluid pressure gradient spontaneously develops across the granular bed. At $t=t_{2}$, we start the rotation of the inner cylinder and mesure the torque $\Gamma$.
In the following, all signal to noise ratios are improved by time averaging the signal over the averaging window (about $5 \mathrm{mn}$ ) represented in Figure 2.b. Note that, over longer time scales (typically hours), very small surface deeping around the rod develops which could originate from slow 3D recirculations ${ }^{25}$.

As shown in Figure 2.c, when the Darcy flow is turned on, $P_{f}$ increases linearly with $z$ and can be well fitted with a line of slope $\nabla P_{f}$. From this measurement and Eqn. 1, the value of coefficient $C$ is then given by

$$
C=\frac{-\nabla P_{f} L}{\rho_{f} g \Delta H} .
$$

Typically, the imposed Darcy pressure gradient for $\Delta H=1 \mathrm{~m}$ corresponds to an effective gravity $g_{\text {eff }} \approx 10 g$. In practice, this means that for measurements performed with $\Delta H$ larger than $0.1 \mathrm{~m}$, the particle pressure is dominated by the effect of the Darcy-flow.

Finally, the value $C$ and the Torque $\Gamma$ give access to the friction coefficient of the suspension using Eqn. 4 and 2. In Figure 2.d, the suspension friction coefficient $\mu$ is plotted for various rotation speeds $\Omega$ and for $\Delta H=0$. For $\Omega<0.1 \mathrm{cps}$, the friction coefficient becomes independent of the rotation speed and is equal to its quasi-static value $\mu_{c}$. Note that for such rotation speeds and assuming the sheared granular layer thickness is about $10 d$ wide $^{22}$, the corresponding viscous number is $J=\eta_{f} a \Omega / 10 d P_{p}<10^{-3}$, well in the quasi-static range ${ }^{19}$.

\section{B. Results}

Systematic experiments were performed for different values of $\Delta H$ (various intensities of the Darcy-flow) and for rotation speeds $\Omega<0.1$ cps to remain in the quasi-static regime. As expected from Eqn. 1, we find that the Darcy pressure gradient $\nabla P_{f}$ increases linearly with the height of the water outlet $\Delta H$ (Fig. 2.e). For each measurement, the Darcy pressure gradient $\nabla P_{f}$ is used to estimate $C$ using Eqn. 5. When varying $\Delta H$, the value of $C$ remains constant with $C \approx 0.63$ (Fig. 2.e, inset). This value of $C$ indicates that over the total pressure $\rho_{f} g \Delta H$ imposed by letting the fluid flow vertically throughout the system, $27 \%$ is balanced by the grid while $63 \%$ is actually acting on the granular bed of particles.

Figure 2.f shows that the torque also increases linearly with $\Delta H$. Using Eqn. 4, the measure of $C$ and of the torque $\Gamma$ give access to the suspension friction coefficient $\mu_{c}$, which is plotted in Figure 2.g versus $\Delta H$ (black horizontal axis) and also versus $\bar{P}_{p}$ (blue horizontal axis), the mean particle pressure: $\bar{P}_{p}=\frac{1}{h} \int_{0}^{h} P_{p}(z) d z$. We find that the suspension friction coefficient $\mu_{c}$ remains constant when the particle pressure $\bar{P}_{p}$ is increased, as expected for a standard Newtonian suspension within the quasi-static regime.

The above results validate the Darcytron concept. Using an external Darcy flow, we were able to control the particle pressure $P_{p}$ in a sheared granular suspension and access the quasistatic suspension friction coefficient $\mu_{c}$. We confirmed that for a standard (non shear-thickening) suspension of macroscopic 
frictional particles, $\mu_{c}$ is independent of the applied pressure, see Figure 2.c. In the following, we will use these results as a benchmark to highlight how the frictional behavior of shearthickening suspensions differs from that of a standard suspension.

\section{APPLICATION OF THE DARCYTRON TO A MODEL SHEAR-THICKENING SUSPENSION}

\section{A. Experimental system}

We now turn to a model shear-thickening suspension composed of non-Brownian silica beads of diameter $d=23.56$ $\mu \mathrm{m}$ and density $\rho_{p}=1850 \mathrm{~kg} / \mathrm{m}^{3}$ immersed in water, see Figure 3.a. We have previously shown that this system exhibits shear thickening at large concentration due to the existence of a short range electrostatic repulsive force in addition to friction ${ }^{13}$. Here we use the Darcytron to probe its frictional behavior under controlled particle pressure. Since the particles are now much smaller, the cell dimensions are scaled down to $25 \mathrm{~mm}$ for its width and $60 \mathrm{~mm}$ for its height. We use the same rough inner cylinder, with radius $a=6.2 \mathrm{~mm}$ and the grid is now made out of a filter cloth with $10 \mu \mathrm{m}$ pores sandwiched between the same two metallic grids. The beads are incorporated into the cell filled with pure water until they form a sediment of height $L=26 \mathrm{~mm}$. Typical signals for the evolution of the fluid pressure and of the torque are shown in Figure 3.d. Here, when the Darcy pressure gradient $\nabla P_{f}$ is measured for a given height of the water outlet, we obtain $C=0.99$. This value of $C$, very close to 1 , indicates that the pressure loss in the grid is negligible compared to that due to the granular sediment.

Compared to the previous standard suspension, the major difference here is that silica particles immersed in water spontaneously develop negative surface charges (Figure 3.b). The resulting charge distribution generates an electrostatic repulsive force $F_{\text {rep }}=F_{0} \exp \left(-r / \lambda_{D}\right)$ between them, where $F_{0}$ is the magnitude of the force, $r$ the distance between the surfaces of the particles and $\lambda_{D}$ the Debye length ${ }^{26-28}$. With this additional force scale, the frictional behavior of the granular pile is expected to change when the particle pressure is varied. At low granular pressure $P_{p}$, the repulsive force can prevent particles from making solid contact: particles thus slide easily against each other without friction. Conversely, when the confining pressure is sufficient to approach the particles at a separation distance smaller than their roughness $\ell_{r}$, particles make solid frictional contact. The critical pressure $P^{*}$ for the onset of this frictional transition is ${ }^{13}$

$$
P^{*}=\frac{4 F_{0}}{\pi d^{2}} e^{-2 \frac{\ell_{r}}{\lambda_{\mathrm{D}}}}
$$

In pure water, $F_{0} / d \simeq 1 \mathrm{mN} \mathrm{m}^{-1}, \lambda_{\mathrm{D}} \simeq 1 \mu \mathrm{m}^{27}$ and AFM measurements of the particle roughness give $\ell_{r}=3.73 \pm 0.80$ nm, see Figure 3.c, which gives $P^{*} \simeq 53 \mathrm{~Pa}$.

In the Darcytron, the particle pressure increases linearly with depth $z$ within the sediment according to $P_{p}(z)=$ $\phi \Delta \rho g_{\text {eff }} z$. There is thus a critical depth $z^{*}=P^{*} / \phi \Delta \rho g_{\text {eff }}(\Delta H)$ at which the confining pressure $P_{p}(z)$ overcomes the repulsive pressure $P^{*}$ : above this critical depth $\left(z<z^{*}\right)$, particles are frictionless and below $\left(z>z^{*}\right)$ they are frictional. This behavior is illustrated in Figure 3.e where the Darcytron is sketched for two extreme values of $\Delta H$. When $\Delta H=0$ (left), the applied pressure is small; the inner cylinder is therefore immersed in a sediment of frictionless particles. Conversely, when $\Delta H=1 \mathrm{~m}$ (right), the applied pressure is large and most of the cylinder is now immersed in a pile of frictional particles.

To probe the frictional transition, we set the immersion height $h$ of the cylinder to $h=1.5 \mathrm{~mm}$ such that, for the smallest applied particle pressure (i.e $\Delta H=0$ ), the cylinder is initially immersed in a sediment of frictionless particles: $h<$ $z^{*}(\Delta H=0) \simeq 10 \mathrm{~mm}$. Under such conditions, the immersed part of the cylinder has an aspect ratio $h / 2 a \approx 0.1$ (recall the cylinder radius is $6.2 \mathrm{~mm}$ ). This low aspect ratio means that the friction of the material beneath the inner cylinder can no longer be neglected compared to that on its lateral vertical side. In the following, we correct the expression of the friction coefficient to account for this extra bottom torque given by $\Gamma_{\text {bottom }}=\int_{0}^{a} 2 \pi r^{2} \mu P_{p}(z=h) d r=\int_{0}^{a} 2 \pi r^{2} \mu \phi \Delta \rho g_{\text {eff }} h d r=$ $2 \pi a^{3} \mu \phi \Delta \rho g_{\text {eff }} h / 3$ (with $K=1$ ). The total torque is thus $\Gamma=\mu \times \pi a^{2} \phi \Delta \rho g_{\text {eff }} h^{2}(1+(2 a / 3 h))$, which leads to the following expression for the suspension friction coefficient:

$$
\mu=\frac{\Gamma}{\pi a^{2} \phi \Delta \rho g_{\mathrm{eff}} h^{2}(1+(2 a / 3 h))} .
$$

\section{B. Evidence of the frictional transition under imposed particle pressure}

Figure 3.f shows the quasi-static friction coefficient $\mu_{c}$ of the silica particle suspension as a function of the particle pressure, which is varied by changing $\Delta H$. Measurements are performed at a rotation speed $\Omega=2 \times 10^{-5} \mathrm{cps}$ to be in quasistatic regime. The main observation is that the friction coefficient here is not constant but gradually increases as the particle pressure $P_{p}$ is increased. At low particle pressure, we find that the friction coefficient of the silica particles suspension is $\mu_{c} \approx 0.1$, which is precisely the expected friction coefficient of a suspension of frictionless particles ${ }^{13,23,24,29,30}$. This result indicates that under low confining pressure the repulsive force prevents solid contact between the silica particles and thus interparticle friction. Upon increasing the confining pressure (by increasing $\Delta H$ ), the friction coefficient is found to gradually increase, meaning that more and more frictional contacts occur within the suspension. For the largest particle pressure investigated $(\Delta H=1 \mathrm{~m})$, we found $\mu_{c} \approx 0.32$, a value approaching that obtained with the previous standard frictional suspension. The above results provide direct evidence that varying the particle pressure induces a frictional transition in this shear thickening suspension, in agreement with the recent models.

We also wish to report that the frictionless state could only be observed for an immersion height of the cylinder $(h=1.5$ $\mathrm{mm}$ ) significantly smaller than the predicted critical depth 
a)

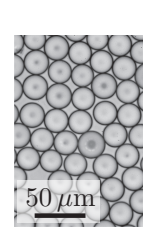

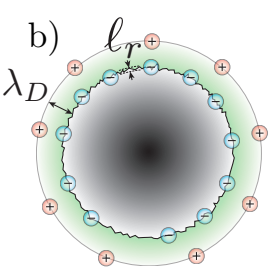

e)

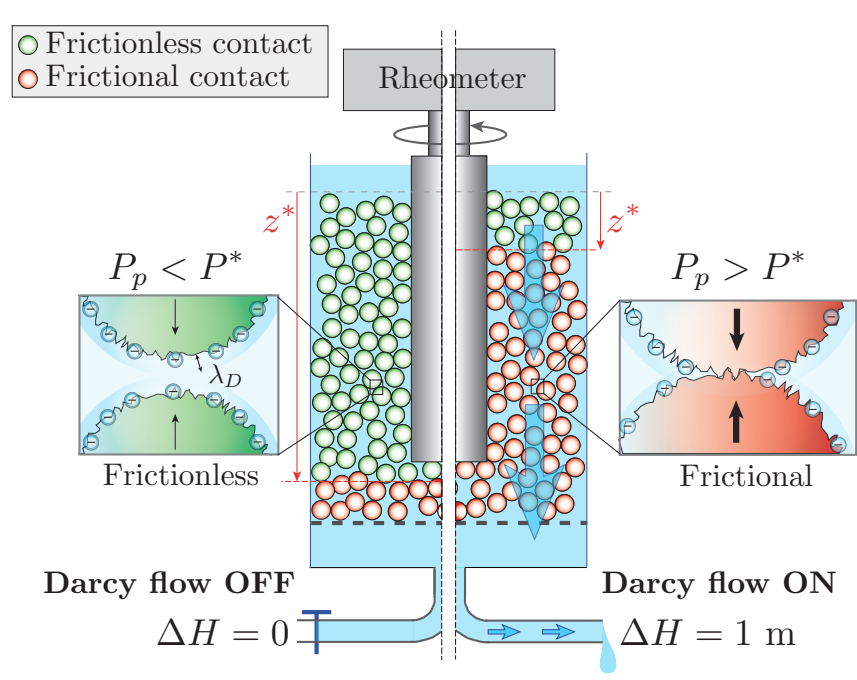

c)

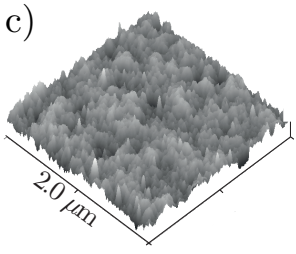

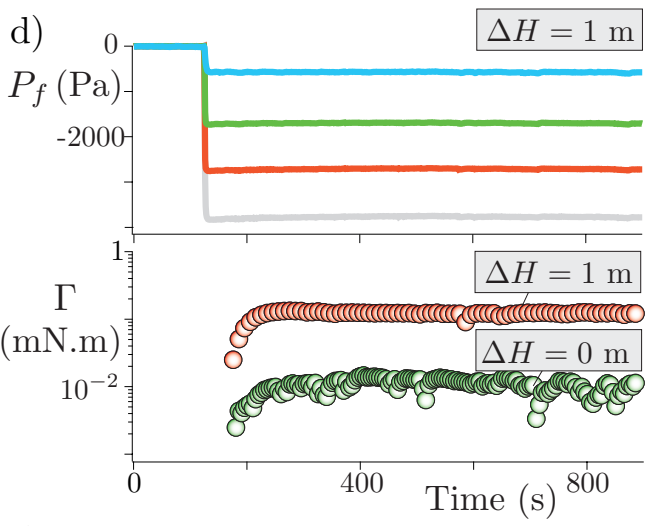

f)

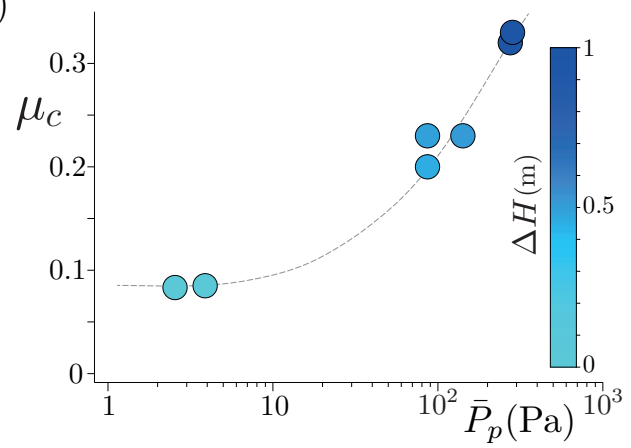

FIG. 3. Application of the Darcytron to a shear-thickening suspension (silica beads $d \approx 25 \mu \mathrm{m}$ immersed in pure water). a) Picture of the silica particles. b) Sketch of a silica particle of roughness $\ell_{r}$ immersed in a ionic aqueous solution. When immersed in water, silica particles spontaneously carry negative surface charges which generate a repulsive force whose range is the Debye lenght $\lambda_{D}$. c) AFM image of the particle surface of roughness $\ell_{r}=3.73 \pm 0.80 \mathrm{~nm}$. d) Fluid pressure and torque versus time for a typical measurement. e) Sketch of half of the Darcytron for $\Delta H=0$ (left) and $\Delta H=1 \mathrm{~m}$ (right). When $\Delta H=0$, the particle pressure is small; the repulsive force prevents particle contacts $\left(P_{p}<P^{*}\right)$ and the inner cylinder is thus immersed in a suspension of frictionless particles. When $\Delta H=1 \mathrm{~m}$, the particle pressure overcome the repulsive force $\left(P_{p}>P^{*}\right)$; most of the cylinder is now immersed in a pile of frictional particles. f) Quasi-static friction coefficient of the suspension $\mu_{c}$ versus average particle pressure $\bar{P}_{p}$.

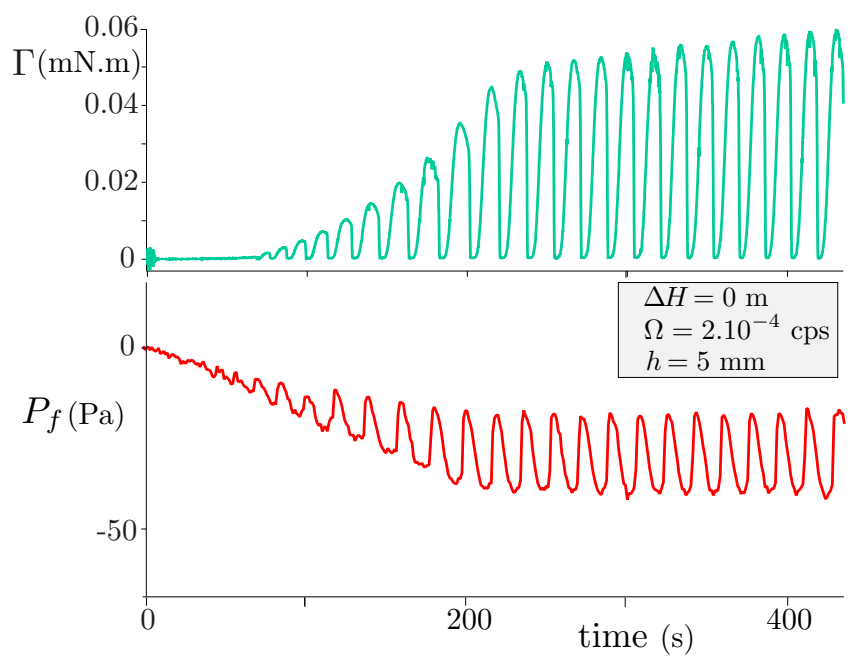

FIG. 4. Unexpected instabilities observed with the silica particles at large immersion depth of the inner cylinder $(h=5 \mathrm{~mm})$. $z^{*}=10 \mathrm{~mm}$. For larger immersion heights, we observed the emergence of unexpected periodic oscillations of both the torque $\Gamma$ and the fluid pressure $P_{f}$, as shown in Figure 4. These oscillations, which are reminiscent of the instabilities observed in many recent studies ${ }^{31-34}$, constitute an intriguing topic for future studies.

\section{Comparison with rotating drum experiments (Clavaud et al. 2017)}

We now compare the results obtained here with the Darcytron to those of Clavaud et al. (2017) ${ }^{13}$, where the frictional transition was highlighted using a rotating drum configuration by investigating the quasi-static avalanche angle $\theta$ of a pile of the same silica particles, see Figure 5.a. The two experiments are complementary. In the Darcytron, the control parameter is the particle pressure $P_{p}$, which is varied within $\bar{P}_{p} \in[4,350]$ Pa by changing $\Delta H$, while the repulsive pressure $P^{*} \simeq 53 \mathrm{~Pa}$ is here fixed and set by the Debye length in pure water. Conversely, in the rotating drum, the confining pressure $\bar{P}_{p}$ cannot be changed as it is set by the weight of the flowing layer of 


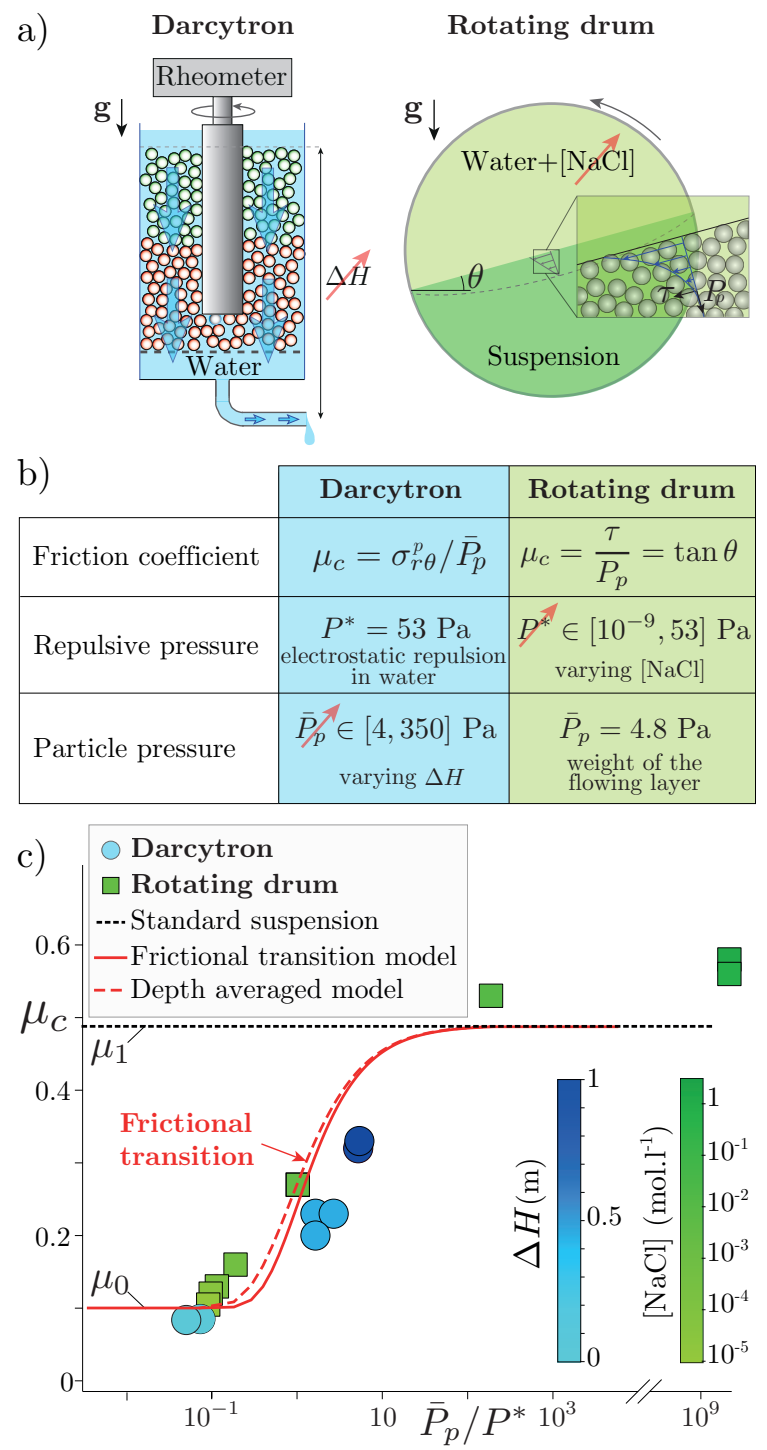

FIG. 5. Comparison with the rotating drum experiments of Clavaud et al. (2017). a) Schematic of the Darcytron (present study) and of the rotating drum ${ }^{13}$ used to investigate the frictional transition in shearthickening suspensions. b) Table showing the complementarity of these two studies. In the Darcytron, the control parameter is $\Delta H$ (blue color bar) varying the particle pressure $\bar{P}_{p}$ while the repulsive pressure, $P^{*}=53 \mathrm{~Pa}$, is fixed. In the rotating drum, the control parameter is the ionic concentration $[\mathrm{NaCl}]$ of the solvent (green color bar) varying the magnitude of the repulsive pressure $P^{*}$ while the particle pressure, $\bar{P}_{p}=4.8 \mathrm{~Pa}$, is fixed. b) Comparison of both studies: quasi-static friction coefficient $\mu_{c}$ of a shear thickening suspension of silica particles versus $\bar{P}_{p} / P^{*}$. Comparison to the frictional behavior of a standard (Newtonian) suspension (black dashed line) and to the frictional transition model (red solid line: local friction coefficient from Equ. 8 with $\mu_{0}=0.1$ and $\mu_{1}=0.49$, red dashed line: depth averaged friction coefficient computed from Equ. 4 and 8).

grains at the top of the pile $\left(\bar{P}_{p}=20 \phi \Delta \rho g d \cos \theta \approx 4.8 \mathrm{~Pa}\right)^{13}$. In that experiment, the control parameter is the ionic concentration $[\mathrm{NaCl}]$ of the solvent which, by changing the Debye
Length $\lambda_{\mathrm{D}}=0.304 / \sqrt{[\mathrm{NaCl}]} \mathrm{nm}\left(\right.$ at $T=25 \mathrm{C}^{\circ}$ ), modifies the magnitude of the repulsive pressure $P^{*}$ (see Eqn. 6). By increasing the salt concentration from $10^{-6}$ to 1 mol. $\mathrm{l}^{-1}$, we changed the repulsive pressure $P^{*}$ from 53 to $10^{-9} \mathrm{~Pa}$. In the table of Figure 5.b, we sum up the parameters and their range of application, highlighting the complementarity of both experiments.

Figure 5.c shows that data obtained both with the Darcytron (varying $\bar{P}_{p}$, keeping $P^{*}$ constant) and with the rotating drum (varying $P^{*}$, keeping $\bar{P}_{p}$ constant) collapse on the same master curve when the quasi-static friction coefficient of the suspension $\mu_{c}$ is plotted as a function of $\bar{P}_{p} / P^{*}$. This collapse confirms that the frictional transition is controlled by a single dimensionless number $\bar{P}_{p} / P^{*}$. Moreover, when compared to the benchmark frictional suspension (dashed line: $\mu_{c}=\mathrm{c}^{\text {te }} \approx 0.5$ ), the results obtained with the silica particles reveal the emergence of the frictionless branch at low values of $\bar{P}_{p} / P^{*}$. This latter observation confirms the fundamental hypothesis of the frictional transition model: that of the existence of a frictionfree state of the suspension under low confining pressure. Note that in Figure 5.c, data with the Darcytron are limited to $\bar{P}_{p} / P^{*}<10$, a much smaller value than with the rotating drum. In order to reach higher $\bar{P}_{p} / P^{*}$ in the Darcytron, one should either apply a larger water pressure drop $\Delta H$, which is limited to $\Delta H \sim 1$ meter in our setup, or decrease the value of the repulsive pressure $P^{*}$ by adding salt in the solution to screen the charges around the silica particles. This last possibility would complement the present work.

In the spirit of Wyart \& Cates model ${ }^{9}$, we propose a simple mixing law to predict how the quasi-static suspension friction coefficient $\mu_{c}$ varies with $P_{p} / P^{*}$ :

$$
\mu_{c}\left(P_{p} / P^{*}\right)=\mu_{1} f+\mu_{0}(1-f),
$$

where $\mu_{0}=0.1$ (resp. $\mu_{1}=0.49$ ) is the quasi-static friction coefficients of the suspension when all contacts are frictionless (resp. frictional) and $f \in[0,1]$ is the fraction of frictional contacts. Figure 5.c shows that the frictional transition observed experimentally is well captured by Eqn. 8 without adjustable parameter when taking $f\left(P_{p} / P^{*}\right)=\exp \left(-P^{*} / \bar{P}_{p}\right)$, as proposed by Wyart \& Cates model ${ }^{9}$. Note that the Darcytron provides an average friction coefficient based on the global torque and the depth averaged pressure (Equ. 4), which may differ from the local friction coefficient. However, we have verified (see Fig. 5.c) that this difference is minor by comparing the local friction coefficient predicted by Equ. 8 (red solid line) to the depth average friction coefficient obtained from Equ. 8 and 4 (red dashed line).

\section{v. DISCUSSION AND CONCLUSION}

An original rheometer based on a new concept, the Darcytron, was designed to study the frictional transition model recently proposed to explain the shear-thickening behavior of certain dense suspensions. The originality of this device is to allow the control of the particle pressure by imposing a vertical Darcy flow across a settled bed of particles. In addition, this rheometer has no lower particle size limit (contrarily to 
that of Boyer et al. ${ }^{14}$ ) since the particle pressure is here not imposed with a moving grid but with a Darcy flow of adjustable intensity.

Our main finding is that the frictional behavior of a shearthickening suspension composed of small silica particles strongly contrasts with that of a standard (Newtonian) suspension of large glass beads, see Figure 5.c. For standard (Newtonian) suspensions, the quasi-static friction coefficient $\mu_{c}$ is independent of the confining pressure $P_{p}$, meaning that the microscopic interparticle friction is independent of the applied stress. This behavior is expected in Newtonian suspensions owing to the absence of other force scales than the shear stress. Conversely, in a shear-thickening suspension of silica particles, the quasi-static friction coefficient is found to increase with the confining pressure. The friction coefficient is found to transit from $\mu_{0}=0.1$ which corresponds to the friction coefficient of a suspension of frictionless particles, when $P_{p} / P^{*} \ll 1$, to $\mu_{1} \approx 0.5$ which corresponds to the friction coefficient of a standard suspension of frictional particles, when $P_{p} / P^{*} \gg 1$. This behavior stems from the presence of an electrostatic repulsive force and its associated repulsive pressure $P^{*}$, which prevents particle contacts and thus friction between particles under low confining pressure. Our experimental results thereby confirm the fundamental hypothesis of the frictional transition model ${ }^{7-9}$ recently proposed to explain shearthickening in certain dense suspensions. Interestingly, our results were obtained in the quasi-static regime where, by definition, measurements are rate-independent (i.e. independent of the rotation speed, shear rate, relative velocity between particles). This suggests that hydrodynamic forces, such as lubrication forces, play no role in the frictional transition and that only the repulsive force prevents the contact between particles in the frictionless state. In this sense, the denomination of the transition as a 'lubricated-to-frictional' transition, which is often found in the literature of shear thickening, is to our opinion misleading.

The new rheological device developed in this study enables the investigation of the rheology of very dense suspensions, where conventional rheology fails as close to jamming, under volume-imposed conditions, stress fluctuations diverge. Moreover, the Darcytron gives direct access to the suspension friction coefficient, a quantity inaccessible to conventional rheology. An interesting perspective would be to extend this technique to be able to explore the full frictional rheology of shear thickening suspensions $(\mu, \phi)$ when varying both the viscous number $J$ and $P_{p} / P^{*}$, thereby exploring all the parameters relevant within the constitutive laws of shear-thickening suspensions. In this case, interesting phenomena such as "negative dynamic compressibility" have been reported from numerical simulations of shear thickening suspensions under imposed pressure ${ }^{35}$. The way the pressure is imposed on the particles (using a Darcy flow) is a new concept in rheometry which may open interesting perspectives both for fundamental research (flow and jamming of dense colloïdal and Brownian suspensions) and for applications such as in the concrete industry.

\section{ACKNOWLEDGMENTS}

We are thankful to Alain Rangis from CINAM for performing the AFM measurements and to the technical staffs at IUSTI for building the experiments. This work was supported by the European Research Council under the European Union Horizon 2020 Research and Innovation programme (ERC grant agreement No. 647384), by the Labex MEC (ANR-10-LABX-0092) under the A*MIDEX project (ANR11-IDEX-0001-02) funded by the French government program Investissements d'Avenir and by ANR ScienceFriction (ANR-18-CE30-0024).

${ }^{1}$ H. Freundlich and H. Röder, "Dilatancy and its relation to thixotropy," Transactions of the Faraday Society 34, 308-316 (1938).

${ }^{2} \mathrm{H}$. Barnes, "Shear-thickening (dilatancy) in suspensions of nonaggregating solid particles dispersed in newtonian liquids," Journal of Rheology 33, 329-366 (1989).

${ }^{3}$ J. F. Morris, "Shear thickening of concentrated suspensions: Recent developments and relation to other phenomena," Annual Review of Fluid Mechanics 52 (2020).

${ }^{4}$ G. Bossis, P. Boustingorry, Y. Grasselli, A. Meunier, R. Morini, A. Zubarev, and O. Volkova, "Discontinuous shear thickening in the presence of polymers adsorbed on the surface of calcium carbonate particles," Rheologica Acta 56, 415-430 (2017).

${ }^{5}$ E. Blanco, D. J. Hodgson, M. Hermes, R. Besseling, G. L. Hunter, P. M. Chaikin, M. E. Cates, I. Van Damme, and W. C. Poon, "Conching chocolate is a prototypical transition from frictionally jammed solid to flowable suspension with maximal solid content," Proceedings of the National Academy of Sciences 116, 10303-10308 (2019).

${ }^{6} \mathrm{~S}$. Arora and A. Ghosh, "Evolution of soft body armor," Advanced Textile Engineering Materials , 499-552 (2018).

${ }^{7}$ R. Seto, R. Mari, J. F. Morris, and M. M. Denn, "Discontinuous shear thickening of frictional hard-sphere suspensions," Idoibase ${ }_{\hookrightarrow}$ 10.1103/PhysRevLett.111.218301 Physical Review Letters 111, 218301 (2013).

${ }^{8}$ R. Mari, R. Seto, J. F. Morris, and M. M. Denn, "Shear thickening, frictionless and frictional rheologies in non-brownian suspensions," Journal of Rheology 58, 1693-1724 (2014).

${ }^{9}$ M. Wyart and M. E. Cates, "Discontinuous shear thickening without inertia in dense non-brownian suspensions," Physical Review Letters 112, 098302 (2014).

${ }^{10}$ J. Comtet, G. Chatté, A. Niguès, L. Bocquet, A. Siria, and A. Colin, "Pairwise frictional profile between particles determines discontinuous shear thickening transition in non-colloidal suspensions," Nature Communications 8, 15633 (2017).

${ }^{11}$ B. M. Guy, M. Hermes, and W. C. Poon, "Towards a unified description of the rheology of hard-particle suspensions," Idoibase _ـ10.1103/PhysRevLett.115.088304 Physical Review Letters 115, 088304 (2015).

${ }^{12}$ N. Y. Lin, B. M. Guy, M. Hermes, C. Ness, J. Sun, W. C. Poon, and I. Cohen, "Hydrodynamic and contact contributions to continuous shear thickening in colloidal suspensions," Idoibase_10.1103/PhysRevLett.115.228304 Physical Review Letters 115, 228304 (2015).

${ }^{13}$ C. Clavaud, A. Bérut, B. Metzger, and Y. Forterre, "Revealing the frictional transition in shear-thickening suspensions," Proceedings of the National Academy of Sciences 114, 5147-5152 (2017).

${ }^{14}$ F. Boyer, É. Guazzelli, and O. Pouliquen, "Unifying suspension and granular rheology," Physical Review Letters 107, 188301 (2011).

${ }^{15}$ S. Dagois-Bohy, S. Hormozi, É. Guazzelli, and O. Pouliquen, "Rheology of dense suspensions of non-colloidal spheres in yield-stress fluids," Journal of Fluid Mechanics 776 (2015).

${ }^{16}$ F. Tapia, O. Pouliquen, and E. Guazzelli, "Influence of surface roughness on the rheology of immersed and dry frictional spheres," Phys. Rev. Fluids 4, 104302 (2019)

${ }^{17}$ R. Jackson, "Locally averaged equations of motion for a mixture of identical spherical particles and a newtonian fluid," Chemical Engineering Science 52, 2457-2469 (1997).

${ }^{18}$ B. Andreotti, Y. Forterre, and O. Pouliquen, Granular media: between fluid and solid (Cambridge University Press, 2013). 
${ }^{19}$ É. Guazzelli and O. Pouliquen, "Rheology of dense granular suspensions," Journal of Fluid Mechanics 852 (2018).

${ }^{20}$ T. Dbouk, L. Lobry, and E. Lemaire, "Normal stresses in concentrated nonBrownian suspensions," \doibase 10.1017/jfm.2012.516 Journal of Fluid Mechanics 715, 239-272 (2013).

${ }^{21}$ M. Depken, J. B. Lechman, M. van Hecke, W. van Saarloos, and G. S. Grest, "Stresses in smooth flows of dense granular media," Europhysics Letters 78, 58001 (2007).

${ }^{22}$ GDRMiDi, "On dense granular flows," The European Physical Journal E 14, 341-365 (2004).

${ }^{23}$ S. Chialvo, J. Sun, and S. Sundaresan, "Bridging the rheology of granular flows in three regimes," Physical Review E 85, 021305 (2012).

${ }^{24}$ M. Trulsson, E. DeGiuli, and M. Wyart, "Effect of friction on dense suspension flows of hard particles,” Physical Review E 95, 012605 (2017).

${ }^{25}$ K. Krishnaraj and P. R. Nott, "A dilation-driven vortex flow in sheared granular materials explains a rheometric anomaly," Nature communications 7 , 10630 (2016).

${ }^{26}$ G. Vigil, Z. Xu, S. Steinberg, and J. Israelachvili, "Interactions of silica surfaces," Journal of Colloid and interface science 165, 367-385 (1994).

${ }^{27}$ J. N. Israelachvili, Intermolecular and surface forces (Academic press, 2011).

${ }^{28}$ V. Valmacco, M. Elzbieciak-Wodka, C. Besnard, P. Maroni, G. Trefalt, and M. Borkovec, "Dispersion forces acting between silica particles across wa- ter: influence of nanoscale roughness," Nanoscale Horizons 1, 325-330 (2016).

${ }^{29}$ P.-E. Peyneau and J.-N. Roux, "Frictionless bead packs have macroscopic friction, but no dilatancy," Physical Review E 78 (2008).

${ }^{30}$ H. Perrin, C. Clavaud, M. Wyart, B. Metzger, and Y. Forterre, "Interparticle friction leads to nonmonotonic flow curves and hysteresis in viscous suspensions," Idoibase _10.1103/PhysRevX.9.031027 Physical Review X 9, 031027 (2019).

${ }^{31}$ B. Saint-Michel, T. Gibaud, and S. Manneville, "Uncovering instabilities in the spatiotemporal dynamics of a shear-thickening cornstarch suspension," Physical Review X 8, 031006 (2018).

${ }^{32}$ R. N. Chacko, R. Mari, M. E. Cates, and S. M. Fielding, "Dynamic vorticity banding in discontinuously shear thickening suspensions," Physical Review Letters 121, 108003 (2018).

${ }^{33}$ J. A. Richards, J. R. Royer, B. Liebchen, B. M. Guy, and W. C. Poon, "Competing timescales lead to oscillations in shear-thickening suspensions,” Physical Review Letters 123, 038004 (2019).

${ }^{34}$ V. Rathee, D. L. Blair, and J. S. Urbach, "Localized transient jamming in discontinuous shear thickening," arXiv preprint arXiv:1908.02856 (2019).

${ }^{35} \mathrm{~J}$. Dong and M. Trulsson, "Analog of discontinuous shear thickening flows under confining pressure,” Physical Review Fluids 2, 081301 (2017). 